\title{
Puberty, Ovarian Steroids, and Stress
}

\author{
ELIZABETH A. YOUNG ${ }^{a}$ AND MARGARET ALTEMUS ${ }^{b}$ \\ ${ }^{a}$ Department of Psychiatry and Mental Health Research Institute, \\ University of Michigan, Ann Arbor, Michigan 48109, USA \\ ${ }^{b}$ Department of Psychiatry, Weill-Cornell Medical School, \\ New York, New York 10021, USA
}

\begin{abstract}
Puberty is accompanied by a number of changes, among them increased risk for development of major depression. The most common etiology of major depression is stressful life events, being present in approximately $90 \%$ of first episodes of depression. The hypothalamic-pituitary-adrenal (HPA) axis is one of the major systems involved in responses to stress, and this system is clearly influenced by ovarian hormones. Normal women demonstrate resistance to negative feedback of both cortisol in the fast-feedback paradigm and dexamethasone in the standard delayed-feedback paradigm. Depressed premenopausal women show greater increases in baseline cortisol than postmenopausal depressed women and than depressed men. Studies in rodents suggest a similar resistance to glucocorticoid feedback but suggest that estradiol can function to inhibit stress responsiveness. Studies of premenopausal depressed women demonstrate lower estradiol, which suggests that there is less inhibitory feedback of estradiol on the HPA axis, while normal progesterone continues to augment stress responses further. The onset of these reproductive hormonal changes modulating stress systems at puberty may sensitize girls to stressful life events, which become more frequent at the transition to puberty and young adulthood.
\end{abstract}

KEYWORDS: ovarian steroids; stress hormones; depression; puberty

\section{INTRODUCTION}

Numerous studies have found that stressful life events play a role in the precipitation of episoles of major depression. ${ }^{1,2}$ Furthermore, the response to stressful life events is influenced by a number of individual characteristics known as "vulnerability." 3 Female gender is one of the clearest constituents of vulnerability. In addition to stress as a precipitant of depression, activation of the main stress hormonal system, the hypothalamic-pituitary-adrenal (HPA) axis is seen in major depression. The HPA axis is sexually dimorphic. In this review, we will examine the influence of ovarian steroids on this axis and then speculate on how this relates to the increased role of depression at puberty.

Address for correspondence: Elizabeth A. Young, Department of Psychiatry and Mental Health Research Institute, University of Michigan, 205 Zina Pitcher Place, Ann Arbor, MI 48109. Voice: 734-936-2087; fax: 734-647-4130.

eayoung@umich.edu

Ann. N.Y. Acad. Sci. 1021: 124-133 (2004). (C) 2004 New York Academy of Sciences. doi: 10.1196/annals.1308.013 
"Stress" initiates a hormonal cascade by releasing corticotropin-releasing factor (CRF), which triggers the release of ACTH from the anterior pituitary corticotrope, which, in turn, triggers the release of adrenal glucocorticoids that feed back at brain and pituitary sites to turn off the stress response. ${ }^{4}$ Studies in both rats and humans suggest that the stress response is sexually dimorphic. Studies in rats and humans suggest that gonadal steroids play an important modulatory role on HPA axis, particularly on sensitivity to glucocorticoid negative feedback. These effects may be on glucocorticoid receptors, on brain corticotropin-releasing hormone (CRH) systems, or on responsiveness to $\mathrm{CRH}$.

Glucocorticoids act via multiple mechanisms at multiple sites of the HPA axis to inhibit their own release. At the pituitary, glucocorticoids have direct effects on POMC gene transcription, POMC mRNA levels, and subsequent ACTH peptide stores in vitro in primary pituitary cultures. ${ }^{5-7}$ These effects involve the classic glucocorticoid receptor (GR, type II), which binds glucocorticoids, is translocated to the nucleus, and binds to sites on the DNA. ${ }^{8}$ Studies have demonstrated that glucocorticoids interact with the $\mathrm{CRH}$ receptors in anterior pituitary, acutely inhibiting the binding of CRH to its receptor and chronically decreasing CRH receptor number. 9,10 Such direct effects of glucocorticoids on $\mathrm{CRH}$ receptors may account for some of the inhibitory action of glucocorticoids on ACTH release in vitro.

In addition to pituitary sites of action, glucocorticoids act at brain sites. Early work by McEwen and colleagues demonstrated a very high-affinity uptake of corticosterone, in the hippocampus of adrenalectomized rats injected in vivo with radiolabelled steroids. ${ }^{11}$ These receptors were difficult to demonstrate in nonadrenalectomized rats, presumably because these sites were saturated under resting conditions. ${ }^{12}$ These receptors were not labeled by $\left[{ }^{3} \mathrm{H}\right]$ dexamethasone, suggesting multiple types of glucocorticoid receptors. ${ }^{13}$ The observation of receptor heterogeneity has been expanded upon by deKloet and colleagues, who have subsequently demonstrated two glucocorticoid receptor types: mineralocorticoid receptor (MR, type I), which has particularly high affinity for the glucocorticoid corticosterone; and glucocorticoid receptor (GR, type II), which preferentially binds dexamethasone. ${ }^{14}$ The type II receptors are widely distributed throughout the brain, while the type I receptors exist predominantly in hippocampus. In addition to action at the pituitary and hypothalamus, there is strong evidence that the hippocampus is the main feedback site in the brain.

\section{THE GLUCOCORTICOID CASCADE HYPOTHESIS}

The importance of hippocampal steroid receptors in feedback regulation of stress has been demonstrated in a number of studies. Studies have demonstrated that removal of the hippocampus leads to increases in anterior pituitary secretion of $\beta$-endorphin in plasma, CRF mRNA, and a limited induction of vasopressin mRNA in parvocellular neurons. ${ }^{15}$ Repeated stress or chronic glucocorticoid administration downregulates hippocampal steroid receptors, but not hypothalamic or pituitary receptors. Animals with downregulated hippocampal glucocorticoid receptors are slow to turn off the corticosterone response to stress, and demonstrate decreased sensitivity to glucocorticoid fast feedback. ${ }^{16}$ This decrease in glucocorticoid receptors and insensitivity to 
negative feedback leads to prolonged hypercortisolism which eventually can result in atrophy in hippocampal neurons and further glucocorticoid hypersecretion. ${ }^{17}$

Glucocorticoid hypersecretion and hippocampal neuronal atrophy are most pronounced in aged rats, a situation analogous to the literature on human depression showing a higher incidence of HPA axis feedback abnormalities in aged individuals. ${ }^{18-20}$ These data provide further support for the hippocampus as a site of glucocorticoid negative feedback.

\section{BASIC STUDIES SUGGESTING SEX DIFFERENCES IN HPA AXIS REGULATION}

Studies in rodents support the existence of sex differences in a number of the elements of the HPA axis. The corticosterone response to stress differs in male and female rats, with female rats demonstrating a greater overall response, faster onset of corticosterone secretion, and a faster rate of rise of corticosterone. A steeper rate of rise is necessary in female rats to elicit glucocorticoid fast feedback. ${ }^{21}$ Corticosterone-binding globulin $(\mathrm{CBG})$ is positively regulated by estrogen and thus higher in female rats; in addition, estrogen and progesterone have been demonstrated to affect the HPA axis independent of the effects of CBG. Chronic estrogen treatment enhances the corticosterone response to stress and delays the recovery from stress in estrogen-treated female rats in comparison to ovariectomized (ovx) female rats. ${ }^{22}$ Estradiol treatment blocked downregulation of hippocampal GRs following chronic administration of RU 28362, a glucocorticoid agonist. Studies by Viau and Meaney ${ }^{23}$ demonstrated a greater ACTH and corticosterone stress response in acute estradiol- treated rats compared with ovx female rats or estradiol plus progesteronetreated female rats after short-term ( $24 \mathrm{~h})$ but not long-term $(48 \mathrm{~h})$ estradiol treatment. A partial estrogen response element is found on the CRH gene, which is able to confer estrogen enhancement of CRH expression in cell cultures, ${ }^{24}$ providing a mechanism by which estradiol may enhance stress responsiveness in females. However, not all studies have demonstrated enhanced stress responsiveness with estradiol treatment of female animals. Studies by Young et al. ${ }^{25}$ and Redei et al. ${ }^{26}$ found that lower doses of estradiol, which produce estradiol concentrations in the physiological range, demonstrated an inhibitory effect of estradiol on HPA axis responsiveness. Similarly, a study by Komesaroff and colleagues ${ }^{27}$ found similar inhibitory effects of physiological doses of estradiol. Furthermore, treatment with estradiol antagonists has been demonstrated to increase stress response. ${ }^{25}$ Consequently, the data in experimental animals is contradictory, but recent studies using physiological doses of estradiol demonstrate inhibitory effects with treatment of several weeks.

Studies of the effect of estradiol in humans have also been contradictory. An early study by the Trier group demonstrated greater stress responsiveness in men than women and also found that treatment of normal males with estradiol led to increased stress responsiveness. ${ }^{28}$ However, treatment of postmenopausal women with estradiol did not result in increased stress responsiveness. ${ }^{29}$ This suggests either sexually dimorphic effects of estradiol or that estradiol treatment of men inhibited testosterone, which is also a potent inhibitory factor on HPA axis responsiveness. Further, a study by Komesaroff et al. ${ }^{30}$ in postmenopausal women demonstrated significant in- 
hibitory effects of 8 weeks of estradiol treatment on both HPA axis and catecholamine response to stress.

Progesterone also affects the HPA axis. In cultured rat hepatoma cells, dexamethasone and progesterone were able to bind to the same receptor, and progesterone was a clear competitive antagonist of dexamethasone binding. Progesterone had a faster binding time than cortisol for the GR, but progesterone binds at a different site on GR than the glucocorticoid binding site. ${ }^{31,32}$ Furthermore, progesterone can increase the rate of dissociation of glucocorticoids from the GR. ${ }^{31}$ Female rats have a greater number of GRs in the hippocampus than do male rats. ${ }^{33}$ Progesterone modulates GR number in hippocampus. ${ }^{34}$ In vivo, progesterone demonstrates antiglucocorticoid effects in intact rats. ${ }^{35}$ While the majority of these effects are exerted at the GR, binding studies from Arriza et al. with expressed human MR demonstrate an affinity of progesterone for MR receptor in a range similar to that of dexamethasone. ${ }^{36}$ Studies by Carey et al. found increase MR binding following progesterone treatment of female rats. ${ }^{37}$

Interactions between gonadal steroids and glucocorticoid feedback in vivo suggest that both estrogen and progesterone may play a role. Both Burgess and $\mathrm{Handa}^{22}$ and Viau and Meaney ${ }^{23}$ have demonstrated that estrogen treatment delays the ACTH and glucocorticoid shut-off following stress in estrogen-treated female rats in comparison to ovx female rats. Following long-term (21 days) estradiol treatment, the potent and selective glucocorticoid RU 28362 was ineffective in blocking etherstress-induced ACTH secretion. Work by Keller-Wood et al. in pregnant ewes and ewes given progesterone infusions found that progesterone can diminish the effectiveness of cortisol feedback on stress responsiveness in vivo, complementing earlier studies demonstrating that progesterone is a GR antagonist in vitro. ${ }^{38}$ Consequently, a number of mechanisms have already been described by which estradiol and progesterone may modulate HPA axis regulation.

\section{SEX DIFFERENCES IN HPA AXIS REGULATION: HUMAN STUDIES}

Given that there are a number of effects of ovarian steroids on the HPA axis, are there differences in HPA axis regulation in normal men and women and could these contribute to the excessive vulnerability to depression in women? We have observed both sex differences in response to CRH and in negative feedback in humans. The lack of a reliable robust stressor until recently has limited studies on sex differences in stress response. Studies by Kirschbaum and Hellhammer have found that oral contraceptives decrease the free cortisol response to a social stressor in women, but that treatment of normal men with estradiol for $48 \mathrm{~h}$ results in enhanced ACTH stress response. ${ }^{28,39} \mathrm{~A}$ recent analysis by the Trier group of all their studies concluded that men show greater ACTH responses than do women to stress, but plasma cortisol levels are not different. ${ }^{39}$ The saliva cortisol response is greatest in men and lutealphase women, while follicular-phase women and women on oral contraceptives show similar responses. ${ }^{39}$ Our studies examining sex differences in response to oCRH found a $40 \%$ greater response in women than men, again consistent with animal studies. Since oCRH is acting at the pituitary, our data support differences at the pituitary and adrenal level, as well as possibly at the level of the CRH neuron. 
Infusion of cortisol "turns off" corticotroph secretion of ACTH and $\beta$ - $\mathrm{LPH} / \beta$-end within $15 \mathrm{~min}$ of the onset of a rise in cortisol in both premenopausal female and agematched male control subjects. Following the termination of the infusion, men exhibited continued inhibition of corticotroph secretion for $60 \mathrm{~min}$, while the women began to secrete $\beta$-LPH/ $\beta$-end within this hour. This difference may be dependent upon progesterone. Women with follicular phase plasma progesterone concentrations (progesterone $=0.26 \pm 0.15 \mathrm{ng} / \mathrm{mL}$ ) exhibited patterns of suppression of $\beta$ $\mathrm{LPH} / \beta$-end secretion similar to the men. However, women with progesterone concentrations typical of the luteal phase (progesterone $=6.85 \pm 0.9 \mathrm{ng} / \mathrm{mL}$ ), showed rebound $\beta$-LPH/ $\beta$-end secretion following termination of cortisol infusion. ${ }^{40}$ These data suggest that progesterone antagonizes the feedback effects of cortisol in humans. These conclusions agree with those of Keller-Wood et al., ${ }^{38}$ demonstrating a similar antagonistic effect of progesterone on the feedback effects of cortisol infusion in ewes. Combined with data demonstrating antagonistic effects of progesterone at the GR, the data suggest that progesterone is an important modulator of HPA axis function.

With respect to the influence of changes in ovarian hormones across the menstrual cycle in women, recent studies by Altemus and colleagues ${ }^{41}$ have found increased resistance to dexamethasone suppression during the luteal phase of the menstrual cycle, as compared to the follicular phase, a change that may again be related to either increased estradiol or progesterone during the luteal phase. Furthermore, ovarian steroids influenced the expression of GR mRNA in lymphocytes, resulting in a decrease in GRs in the luteal phase compared to the follicular phase of the menstrual cycle $^{41}$ and suggesting that decreases in GRs may explain the decreased response to dexamethasone. In a design that allows investigators to distinguish the effects of progesterone from those of estrogen, Roca and coworkers ${ }^{42,43}$ studied control women treated first with Lupron, a gonadotrophin-releasing hormone ( $\mathrm{GnRH})$ agonist, which causes suppression of both estrogen and progesterone secretion, and then given sequential replacement of the two hormones. They examined the response to exercise stress as well as to dexamethasone feedback and found that the exercise stress response was increased, and response to dexamethasone feedback was decreased, during the progesterone "add back" phase but not during the estrogen add-back phase. Again, these data suggest that progesterone acts as a glucocorticoid antagonist. Thus, the data from human studies suggest that ovarian steroids, and in particular progesterone, influence the HPA axis response to stress by modulating sensitivity to negative feedback. Furthermore, some data suggest that progesterone may have negative effects on mood particularly in women with premenstrual dysphoric disorder (PMDD) in which depressive symptoms occur in the luteal phase of the menstrual cycle when progesterone levels are high. Although the exact role of sex hormones in this disorder has not been established, estrogen and progesterone suppression by Lupron has been reported to produce significant symptom improvement in depression in PMDD. ${ }^{43}$

In humans, pregnancy is accompanied by increases in both estrogen and progesterone and thus provides another model to examine the interactions between gonadal steroids and the HPA axis. Increases in plasma CBG and cortisol during pregnancy are well known. In humans, dexamethasone challenge studies indicate resistance to glucocorticoid negative feedback during pregnancy. ${ }^{4-46}$ The degree to which higher levels of CBG-bound cortisol contribute to the dexamethasone resistance, as mani- 
fested by higher levels of plasma cortisol following dexamethasone administration, is not completely known. Although dexamethasone itself is not bound by CBG, pregnancy could alter the metabolism of dexamethasone, resulting in less dexamethasone bioavailability. However, at least one study ${ }^{46}$ demonstrated higher free cortisol during pregnancy, higher free-cortisol production following an ACTH infusion, decreased suppressibility of free cortisol by dexamethasone, and a normal circadian rhythm of cortisol, pointing to a change in cortisol set-point during pregnancy. These data are compatible with the data of Keller-Wood demonstrating that high circulating levels of progesterone can antagonize the effects of glucocorticoids on negative feedback. 38

From these studies, it is clear that ovarian steroids do play a modulatory role on HPA axis regulation. However, we do not know which ovarian steroids are involved and which levels of the HPA axis are affected; the underlying mechanisms are not fully elucidated.

\section{SEX DIFFERENCES IN HPA AXIS REGULATION IN DEPRESSION}

Morning and evening cortisol hypersecretion. Our studies examining baseline cortisol secretion in the morning in 16 depressed patients and 16 age- and sex-matched control patients found increased cortisol secretion in the group as a whole, as would be expected. However, there were clear sex differences. While the male patients and their matched controls demonstrated the same plasma cortisol concentration, the female depressed patients demonstrated significantly higher mean plasma cortisol concentration $(11.3 \pm 0.9 \mu \mathrm{g} / \mathrm{dL})$ than their matched controls $(8.1 \pm 0.95 \mu \mathrm{g} / \mathrm{dL}$; significant by a two-tailed $t$-test, $P=0.033$ ). Removal of glucocorticoid negative feedback by metyrapone demonstrates increased central drive in depressed patients in the evening. The response to metyrapone demonstrated sex differences; only the female depressed patients manifested rebound $\beta$ - $\mathrm{LPH} / \beta$-end secretion in comparison to their matched controls (ANOVA, $\mathrm{F}=8.8, \mathrm{df}=1, P=.01$ ); the males did not.

Cortisol hypersecretion and dexamethasone nonsuppression. Other studies examined whether the loss of gonadal steroids at menopause impacts HPA axis regulation in depressed women. We conducted studies using a protocol examining baseline and post-dexamethasone secretion of $\beta$-LPH/ $\beta$-end and cortisol over the course of the day (8 A.M.-4 P.M.). We conducted these studies on 51 depressed women; 36 were premenopausal and 15 were postmenopausal. The premenopausal women demonstrated a significantly lower incidence of pituitary $(\beta-\mathrm{LPH} / \beta$-end) nonsuppression $(n$ $=36$; Nonsuppressor $=44 \%)$ than the postmenopausal women $(n=15$; Nonsuppressor $=81 \%$ ). To determine which of a number of potential variables were associated with $\beta$-LPH/ $\beta$-end nonsuppression in women, a stepwise regression analysis was done. Independent variables included: age, menopausal status, baseline $\beta-\mathrm{LPH} / \beta$ end and cortisol, severity of depression as assessed by Hamilton Depression rating scores, and the number of previous episodes of depression. The dependent variable was $\beta$-LPH/ $\beta$-end nonsuppression. While age had a significant effect on $\beta$-LPH/ $\beta$ end nonsuppression, menopausal status was also important and, combined with cortisol, gave a correlation coefficient of 0.817 . This suggests that menopausal status, in conjunction with cortisol hypersecretion, is a critical variable in the development 
of HPA dysregulation, as manifested by resistance to dexamethasone, and accounts for $65 \%$ of the variance. Further, the lower rate of $\beta$-LPH/ $\beta$-end nonsuppression in premenopausal women suggests that gonadal steroids may modulate the HPA axis and exert some protective effect against high levels of endogenous glucocorticoids (cortisol).

The formulation of the glucocorticoid cascade hypothesis by Sapolsky suggested that stress and repeated bouts of hypercortisolemia lead to downregulation of glucocorticoid receptors, which in turn results in further glucocorticoid hypersecretion, eventually leading to loss of hippocampal neurons, that is, a "glucocorticoid feed forward cascade." ${ }^{\prime 17}$ His studies in rats have suggested that aging is a critical variable and that aging rats demonstrate downregulation of GRs, failure to shut off stress-induced glucocorticoid secretion and hippocampal neuronal loss. Aging is also associated with HPA axis dysregulation in depression. ${ }^{18-20}$ We were very interested whether recurrent depressive episodes with recurrent episodes of hypercortisolemia would lead to progressive HPA axis dysregulation. We divided patients into first episode vs. recurrent unipolar depression, and examined differences in rates of pituitary nonsuppression. We found no association of $\beta$-LPH/ $\beta$-end nonsuppression with recurrent episodes, but within the recurrent and first-episode groups, older age was associated with a higher incidence of HPA axis dysregulation. We also used the absolute number of episodes as a continuous variable and then examined baseline hormonal measures and post-dexamethasone hormonal measures as continuous variables or nonsuppression as a categorical variable. Again, the data did not support the hypothesis that recurrent episodes of depression were associated with progressive HPA axis dysregulation, but rather that aging was the critical variable. Since 16 of the 20 subjects over 50 were women, we cannot determine whether aging is also a factor in men. However, aging is more important than absolute number of episodes in women, and, the previous analysis suggested that menopausal status was the critical variable in aging.

In summary, menopause itself is not associated with increases in plasma cortisol concentrations, but it is associated with an increase in dexamethasone resistance in depressed women. Resistance to dexamethasone suppression is strongly associated with increased baseline cortisol secretion, and thus appears to reflect the development of GR downregulation following a period of hypercortisolemia. That premenopausal women demonstrate less resistance to dexamethasone suppression suggests that they are more resistant to endogenous glucocorticoids and to GR downregulation.

\section{SPECULATIONS ABOUT PUBERTY}

The finding of an increased ACTH response to stress has very important implications for our understanding of stress and stress responsiveness in human females. It is clear that a number of diseases that have been linked to "stress" are more common in females, including depression and other anxiety disorders. Since females demonstrate resistance to negative feedback effects of glucocorticoids; ${ }^{40,41,47}$ this suggests an exaggerated central $\mathrm{CRH}$ response to stress, ${ }^{48}$ which may explain some of the susceptibilty of females to depression and other stress-related disorders. Additionally, resistance to endogenous glucocorticoids may contribute to the increased inci- 
dence of depression. If we believe Munck's hypothesis ${ }^{49}$ that the purpose of glucocorticoids is to turn off not just the HPA axis to stress, but the entire stress response, then this resistance to glucocorticoids would further exaggerate stress responsiveness in women. Studies by Frank and colleagues clearly demonstrate that stressful life events play a role in the precipitation of depression in adolescents. ${ }^{50} \mathrm{As}$ girls reach puberty, these known effects of ovarian steroids on stress systems come into play. Recent studies suggest that elevated glucocorticoids can inhibit autonomic nervous system response, ${ }^{51}$ supporting a role for glucocorticoids in terminating stress-induced activation of the autonomic stress system. This resistance to glucocorticoids may also lead to increasing levels of anxiety at puberty in girls, which may serve to "drive" the onset of depression. ${ }^{52}$ There is evidence that the increased vulnerability to depression in women arises at puberty, when gonadal steroids would further enhance stress responsiveness. Some data suggest that following menopuase, the incidence of depression begins to decrease in women, perhaps as a consequence of increasing sensitivity to glucocorticoids. Additionally, depressed adult women demonstrate lower estradiol, which means that the inhibitory effects of estradiol on stress responsiveness are less. ${ }^{53}$ We would expect that adolescent girls may be in a similar situation. Finally, while most effects of estradiol on serotonin systems are beneficial, decreases in 5HT $1 \mathrm{~A}$ terminal receptors may further sensitize to depression. $^{54}$

\section{REFERENCES}

1. Brown, G.W. \& T. Harris. 1978. Social Origins of Depression: A Study of Psychiatric Disorder in Women. The Free Press. New York.

2. Frank, E., B. Anderson, C. Reynolds, et al. 1994. Life events and the research diagnostic criteria endogenous subtype: a confirmation of the distinction using the Bedford College methods. Arch. Gen. Psychiatry 51: 519-524.

3. Kendler, K.S., R.C. Kessler, E.E. WAlters, et al. 1995. Stressful life events, genetic liability and onset of an episode of major depression in women. Am. J. Psychiatry 152: 833-842.

4. Keller-Wood, M.E. \& M.F. Dallman. 1985. Corticosteroid inhibition of ACTH secretion. Endocrinol. Rev. 5: 1-24.

5. Schacter, B.S., L.K. Johnson, J.D. Baxter \& J.L. Roberts. 1982. Differential regulation by glucocorticoids of proopiomelanocortin mRNA levels in the anterior and intermediate lobes of the rat pituitary. Endocrinology 110: 1142.

6. Roberts, J.L., M.L. Budarf, J.D. Baxter \& E. Herbert. 1979. Selective reduction of proadrenocorticotropin/endorphin proteins and messenger ribonucleic acid activity in mouse pituitary tumor cells by glucocorticoids. Biochemistry 18: 4907-4915.

7. Birnberg, N.C., O. Civelli, J.C. Lissitzski, et al. 1982. Regulation of proopiomelanocortin gene expression in the pituitary and central nervous system. Endocrinology 110: 134A.

8. SAKLY, M. \& B. KoCH. 1981. Ontogenesis of glucocorticoid receptors in anterior pituitary gland: transient dissociation among cytoplasmic receptor density, nuclear uptake and regulation of corticotropic activity. Endocrinology 108: 591.

9. Childs, G.V., J.L. Morell, A. Niendorf \& G. Aguilera. 1986. Cytochemical studies of corticotropin releasing factor receptors in anterior lobe corticotrophs: binding, glucocorticoid regulation and endocytosis of [biotinyl-ser1] CRF. Endocrinology 119: 2129.

10. Schwartz, J., N. Billestrup, M. Perrin, et al. 1986. Identification of corticotropin releasing factor target cells and effects of dexamethasone on binding in anterior pituitary using a flourescent analog of CRF. Endocrinology 119: 2376. 
11. McEwen, B.S., J.M. Weiss \& L.S. Schwartz. 1968. Selective retention of corticosterone by limbic structures in the rat brain. Nature 220: 911.

12. McEwen, B.S., J.M. Weiss \& L.S. Schwartz. 1970. Retention of corticosterone by cell nuclei from brain regions of adrenalectomized rats. Brain Res. 17: 471.

13. DeKloet, R., G. Wallach \& B.S. McEwen. 1975. Differences in corticosterone and dexamethasone binding to rat brain and pituitary. Endocrinology 96: 598.

14. Reul, J.M.H \& E.R. DEKLOET. 1985. Two receptor systems for corticosterone in rat brain: microdistribution and differential occupation. Endocrinology 117: 2505-2511.

15. Herman, J.P., M.K-H. Schafer, E.A. Young, et al. 1989. Hippocampal regulation of the hypothalamo-pituitary-adrenocortical axis: in situ hybridization analysis of CRF and vasopressin messenger RNA expression in the hypothalamic paraventricular nucleus following hippocampectomy. J. Neurosci. 9: 3072-3082.

16. Young, E.A. \& D. VAzQuez. 1996. Hypercortisolemia, hippocampal glucocorticoid receptors and fast feedback. Mol. Psychiatry 1: 149-159.

17. SAPolsky, R.M., L.C. KReY \& B.S. McEwen. 1986. The neuroendocrinology of stress and aging: the glucocorticoid cascade hypothesis. Endocrinol. Rev. 7: 284-301.

18. Halbreich, U., G.M. Asnis, B. Zumoff \& R.S. Nathan. 1984. The effect of age and sex on cortisol secretion in depressives and normals. Psychiatry Res. 13: 221-229.

19. Lewis, D.A., B. Pfohl, J. Schlecte \& W. Coryell. 1984. Influence of age on the cortisol response to dexamethasone. Psychiatry Res. 13: 213-220.

20. Akil, H., R. Haskett, E.A. Young, et al. 1993. Multiple HPA profiles in endogenous depression: effect of age and sex on cortisol and beta-endorphin. Biol. Psychiatry 33: $73-85$.

21. Jones, M.T., F.R. Brush \& R.L.B. Neame. 1972. Characteristics of fast feedback control of corticotrophin release by corticosteroids. J. Endocrinol. 55: 489.

22. Burgess, L.H. \& R.J. HANDA. 1992 . Chronic estrogen-induced alterations in adrenocorticotropin and corticosterone secretion, and glucocorticoid receptor-mediated functions in female rats. Endocrinology 131: 1261-1269.

23. Viau, V. \& M.J. Meaney. 1991. Variations in the hypothalamic-pituitary-adrenal response to stress during the estrous cycle in the rat. Endocrinology 129: 2503-2511.

24. Vamvakopoulos, N.C. \& G.P. Chrousos. 1993. Evidence of direct estrogenic regulation of human corticotropin-releasing hormone gene expression. J. Clin. Invest. 92: 1896-1902.

25. Young, E.A., M. Altemus, V. Parkison \& S. Shastry. 2001. Effects of estrogen antagonists and agonists on the ACTH response to restraint stress. Neuropsychopharmacology 25: 881-891.

26. Redei, E., L. Li, R.F. McGivern \& F. Aird. 1994. Fast glucocorticoid feedback inhibition of ACTH secretion in the ovariectomized rat: effect of chronic estrogen and progesterone. Neuroendocrinology 60: 113-123.

27. Komesaroff, P.A., M. Esler, I.J. Clarke, et al. 1998. Effects of estrogen and estrous cycle on glucocorticoid and catecholamine responses to stress in sheep. Am. J. Physiology 275: E671-E678.

28. Kirschbaum, C., N. Schommer, I. Federenko, et al. 1996. Short-term estradiol treatment enhances pituitary-adrenal axis and sympathetic responses to psychosocial stress in healthy young men. J. Clin. Endocrinol. Metab. 81: 3639-3643.

29. Kudielka, B.M., A.K. Schmidt-Reinwald, D.H. Hellhammer \& C. Kirschbaum. 1999. Psychological and endocrine responses to psychosocial stress and dexamethasone/corticotropin-releasing hormone in healthy postmenopausal women and young controls: the impact of age and a two-week estradiol treatment. Neuroendocrinology 70: $422-430$.

30. Komesaroff, P.A., M.D. Esler \& K. Sudhir. 1999. Effects of estrogen on stress responses in women. J. Clin. Endocrinol. Metab. 84: 4292-4293.

31. Rousseau, G.G., J.D. Baxter \& G.M. Tomkins. 1972. Glucocorticoid receptors: relations between steroid binding and biological effects. Mol. Biol. 67: 99-115.

32. SVEC, F. 1988. Differences in the interaction of RU 486 and ketoconazole with the second binding site of the glucocorticoid receptor. Endocrinology 123: 1902-1906.

33. Turner, B.B. \& D.A. WeAver. 1985. Sexual dimorphism of glucocorticoid binding in rat brain. Brain Res. 343: 16-23. 
34. Ahima R.S., A.N.L. Lawson, S.Y.S. Osei \& R.E. Harlan. 1992. Sexual dimorphism in regulation of type II corticosteroid receptor immunoreactivity in the rat hippocampus. Endocrinology 131: 1409-1416.

35. Duncan, M.R. \& G.R. Duncan. 1979. An in vivo study of the action of antiglucocorticoids on thymus weight ratio, antibody titre and the adrenal-pituitary-hypothalamus axis. J. Steroid Biochem. 10: 245-259.

36. Arriza, J.L., C. Weinberger, G. Cerelli, et al. 1987. Cloning of human mineralocorticoid receptor complementary DNA: structural and functional kinship with the glucocorticoid receptor. Science 237: 268-275.

37. Carey, M.P., C.H. Deterd, J. De Koning, et al. 1995. The influence of ovarian steroids on hypothalamic-pituitary-adrenal regulation in the female rat. J. Endocrinology 144: $311-321$.

38. Keller-Wood, M., J. Silbiger \& C.E. Wood. 1988. Progesterone attenuates the inhibition of adrenocorticotropin responses by cortisol in nonpregnant ewes. Endocrinology 123: 647-651.

39. Kirschbaum, C., B.M. KudielKa, J. GaAB, et al. 1999. Impact of gender, menstrual cycle phase, and oral contraceptives on the activity of the hypothalamic-pituitaryadrenal axis. Psychosom. Med. 61: 154-162.

40. Young, E.A. 1995. The glucocorticoid cascade hypothesis revisited: role of gonadal steroids. Depression 3: 20-27.

41. Altemus, M., L. Redwine, L. Yung-Mei, et al. 1997. Reduced sensitivity to glucocorticoid feedback and reduced glucocorticoid receptor mRNA expression in the luteal phase of the menstrual cycle. Neurosychopharmacology 17: 100-109.

42. Roca, C.A., P.J. Schmidt, M. Altemus, et al. 1998. Effects of reproductive steroids on the hypothalamic-pituitary-adrenal axis response to low dose dexamethasone. Abstract at Neuroendocrine Workshop on Stress. New Orleans, LA.

43. Roca, C.A., P.J. Schmidt, M. Altemus, et al. 2003. Differential menstrual cycle regulation of hypothalamic-pituitary-adrenal axis in women with premenstrual syndrome and controls. J. Clin. Endocrinol. Metab. 88: 3057-3063.

44. Demey-Ponsart, E., J.M. Foidart, J. Sulon \& J.C. Sodoyez. 1982. Serum CBG, free and total cortisol and circadian patterns of adrenal function in normal pregnancy. J. Steroid Biochem. 16: 165-169.

45. CARR, B.R., C.R. PARKer, JR., J.D. Madden, et al. 1981. Maternal plasma adrenocorticotropin and cortisol relationships throughout human pregnancy. Am. J. Obstet. Gynecol. 139: 416-422.

46. Nolten, W.E. \& P.A. Rueckert. 1981. Elevated free cortisol index in pregnancy: possible regulatory mechanisms. Am. J. Obstet. Gynecol. 139: 492-498.

47. Young, E.A. 1996. Sex differences in response to exogenous corticosterone. Mol. Psychiatry 1: 313-319.

48. Altemus, M., C. Roca, E. Galliven, et al. 2001. Increased vasopressin and adrenocorticotropin responses to stress in the midluteal phase of the menstrual cycle. J. Clin. Endocrinol. Metab. 86: 2525-2530.

49. MunCK, A. \& P.M. GuYRE. 1986. Glucocorticoid physiology, pharmacology and stress. Adv. Exp. Med Biol. 196: 81-96.

50. Cyranowski, J.M., E. Frank, E.A. Young \& M.K. Shear. 2000. Adolescent onset of the gender difference in lifetime rates of major depression: a theoretical model. Arch. Gen. Psychiatry 57: 21-27.

51. McEwen, B. 1995. Adrenal steroid actions on brain dissecting the fine line between protection and damage. In Neurobiological and Clinical Consequences of Stress: From Normal Adaptation to PTSD. M.J. Friedman, D.S. Charney \& A.Y. Deutch, Eds.: 135-147. Lippincott-Raven Publishers. Philadelphia.

52. Breslau, N., H. Chilcoat \& L.R. Schultz. 1998. Anxiety disorders and the emergence of sex differences in major depression. J. Gender Specif. Med. 1: 33-39.

53. Young, E.A., A.R. Midgley, N.E. Carlson \& M.B. Brown. 2000. Alteration in the hypothalamic-pituitary-ovarian axis in depressed women. Arch. Gen. Psychiatry 57: 1157-1162.

54. Lu, N.Z. \& C.L. BetheA. 2002. Ovarian steroid regulation of 5-HT1A receptor binding and $G$ protein activation in female monkeys. Neuropsychopharmacology 27: 12-24. 